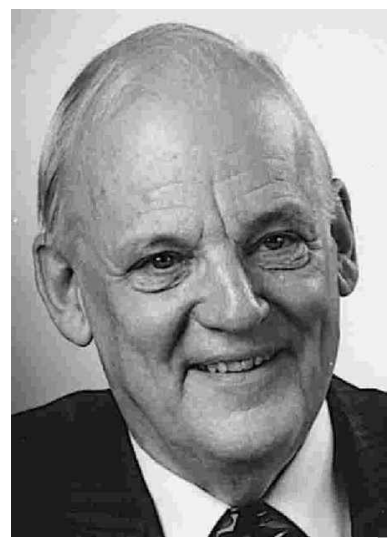

Gösta Samuelson gosta.samuelson@htu.se

\title{
Scandinavian Journal of Nutrition goes international
}

$\mathrm{T}$ The Scandinavian Journal of Nutrition (SJN) now appears in a new form. During the past few years the number of original and review articles in English, as well as the special section in Swedish, have increased. To find new ways in which to publish and market the journal internationally we began a collaboration with the well-known publisher Taylor \& Francis on 1 January 2002.

As before, the first part of every issue will contain review articles and commentaries, followed by original publications, all in English. The second section contains articles in Swedish focusing on top news, lectures from conferences suitable for education in nutrition, and commentaries on current debates in nutrition, particularly concerning functional foods.

A new editorial board will be elected during the year, and the editorial office has been strengthened by the addition of four well-known scientists. Other news is that readers can access SJN both on paper and through electronic media. Subscribers to the journal will have full access to all articles on the web, at www.tandf.no/nutrition.

We hope that our readers will appreciate these changes.

\section{Dietary compounds regulate gene expression}

Several dietary compounds regulate gene expression in the same manner as steroid hormones. This means that nuclear receptors bind dietary substances or their metabolites. Eva Enmark and JanÅke Gustafsson present the state of the art in a review article concerning nuclear receptors, which regulate gene expression by binding ligands such as steroid and thyroid hormones, intermediate steroids, bile acid metabolites and environmental pollutants. Cholesterol and steroid metabolites are nuclear receptor ligands. In their article different nuclear receptors and their roles are presented and possible mechanisms in future clinical uses are discussed.

Furthermore, dietary fatty acids, which are important for growth and cell membranes, have been identified as transcription factors. A novel subtype of oestrogen receptor is expressed in different tissues. The receptor also plays a role in diets rich in phyto-oestrogens, e.g. from soy products.

\section{New aspects on infant iron-deficiency anaemia}

Very few up-to-date studies have been published which compare iron status in infants born in developing countries with infants born at the same time in developed countries. Recently, a randomized study was performed by Domellöf et al. in Honduras and Sweden. Iron-unsupplemented Honduran and Swedish infants, all exclusively breastfed until 6 months and with normal birth weight, were studied until 9 months of age. The results are referred to in a review article by Magnus Domellöf and Olle Hernell. In the Honduran infants a higher prevalence of iron-deficiency anaemia (IDA) was already found at 4 months of age, and when the infants were partially breast-fed the Honduran infants had a much higher prevalence of IDA at 9 months compared with the Swedish infants. Poor nutritional status of the mothers and limited foetal iron accretion may explain these differences. New cut-off limits for haemoglobin ( $\mathrm{Hb}$ ) are suggested in the article, based on the results of the studied iron-replete, breast-fed, unsupplemented infants at 4-9 months of age. The authors conclude that iron requirements should not be based on laboratory values or theoretical calculations. Instead, recommendations should be based on the minimal iron intake that prevents the physiological consequences of IDA. Further randomized, well-controlled studies of minimal iron intake are needed. 